\title{
Parental genetic material and oxygen concentration affect hatch dynamics of mouse embryo in vitro
}

Shaoquan Zhan ${ }^{1 \dagger}$, Shanbo Cao ${ }^{1,2 \dagger}$, Hongzi Du', Yuan Sun', Li Li ${ }^{1}$, Chenhui Ding ${ }^{3}$, Haiyan Zheng ${ }^{1 *}$ and Junjiu Huang ${ }^{1,3^{*}}$

\begin{abstract}
Background: Hatching is crucial for mammalian embryo implantation, since difficulties during this process can lead to implantation failure, ectopic pregnancy and consequent infertility. Despite years of intensive researches, how internal and external factors affecting embryo hatch are still largely unclear.

Methods: The effects of parental genetic material and oxygen concentration on hatch process were examined. Fertilized and parthenogenetic mouse preimplantation embryos were cultured in vitro under 5 and $20 \%$ oxygen for 120 h. Zona pellucida drilling by Peizo micromanipulation were performed to resemble the breach by sperm penetration.

Results: Firstly, parthenogenetic embryos had similarly high blastocyst developmental efficiency as fertilized embryos, but significantly higher hatch ratio than fertilized embryos in both $\mathrm{O}_{2}$ concentrations. $5 \% \mathrm{O}_{2}$ reduced the hatch rate of fertilized embryos from 58.2 to $23.8 \%$, but increased that of parthenogenetic embryos from 81.2 to $90.8 \%$ significantly. Analogously, $5 \% \mathrm{O}_{2}$ decreased the ratio of Oct4-positive cells in fertilized blastocysts, whereas increased that in parthenogenetic blastocysts. Additionally, $5 \% \mathrm{O}_{2}$ increased the total embryonic cell number in both fertilized and parthegenetic embryos, when compared to $20 \% \mathrm{O}_{2}$, and the total cell number of fertilized embryos was also higher than that of parthegenetic embryos, despite $\mathrm{O}_{2}$ concentration. Real-time PCR revealed that the expression of key genes involving in MAPK pathway and superoxide dismutase family might contribute to preimplantation development and consequent blastocyst hatch in vitro. Finally, we showed that fertilized and parthenogenetic embryos have diverse hatch dynamics in vitro, although the zona pellucida integrity is not the main reason for their mechanistic differences.

Conclusion: Both parental genetic material and $\mathrm{O}_{2}$ concentration, as the representative of intrinsic and extrinsic factors respectively, have significant impacts on mouse preimplantation development and subsequent hatch dynamics, probably by regulating the gene expression involving in MAPK pathway and superoxide dismutase family to control embryonic cell proliferation and allocation of ICM cells.
\end{abstract}

Keywords: Hatch, Fertilized embryo, Parthenogenetic embryo, Oxygen, Zona pellucida

\footnotetext{
*Correspondence: ceozhy_1985@126.com; hjunjiu@mail.sysu.edu.cn

${ }^{\dagger}$ Equal contributors

${ }^{1}$ Center for Reproductive Medicine, Key Laboratory for Reproductive Medicine of Guangdong Province, Key Laboratory for Major Obstetric Diseases of Guangdong Province, and Key Laboratory for Reproduction and Genetics of Guangdong Higher Education Institutes, the Third Affiliated Hospital of Guangzhou Medical University, Guangzhou 510150, China Full list of author information is available at the end of the article
}

(c) The Author(s). 2018 Open Access This article is distributed under the terms of the Creative Commons Attribution 4.0 International License (http://creativecommons.org/licenses/by/4.0/), which permits unrestricted use, distribution, and reproduction in any medium, provided you give appropriate credit to the original author(s) and the source, provide a link to the Creative Commons license, and indicate if changes were made. The Creative Commons Public Domain Dedication waiver (http://creativecommons.org/publicdomain/zero/1.0/) applies to the data made available in this article, unless otherwise stated. 


\section{Background}

Hatching is a process essential for mammalian embryo implantation, in which the embryo at blastocyst stage escapes from its zona pellucida (ZP), an outer shell which is composed of glycoproteins and is responsible for the preventation of polyspermy and ectopic pregnancy [1-3]. The mechanism regulating the preimplantation embryos when and where to hatch is very critical for implanting in uterus exactly and successfully, since difficulties during this process can lead to implantation failure, ectopic pregnancy and consequent infertility $[4,5]$.

Many factors have been proved to function in embryo implantation in the past few decades, including embryonic cell number, trypsin-like proteinases, progranulin, prostanoid pathways, uterine function and the leukaemia inhibitory factor, zygote genomic expression and epigenetic changes, such as DNA methylation and histone deacetylation [6-14]. However, none of them has been identified as the determinant factor for embryo hatching. Additionally, the external culture environment is regarded as a key factor for preimplantation embryo growth in vitro, one of which is the oxygen $\left(\mathrm{O}_{2}\right)$ concentration [15]. $\mathrm{O}_{2}$ plays an important role in the regulation of embryonic gene expression, allocation of cells to inner cell mass (ICM) and trophectoderm (TE) lineages and subsequent fetal development $[16,17]$. Recent studies have showed that low $\mathrm{O}_{2}$ (5\%) can promote embroyonic cell proliferation and reduce apoptosis, whereas it inhibit trophoblast cell invasion [17-25]. Conversely, other studies argued that there are no beneficial effects of $5 \% \mathrm{O}_{2}$ culture on embryo subsequent development, such as implantation rate, pregnancy rate and fetal weight, when compared with atmospheric $\mathrm{O}_{2}$ culture $[19,26,27]$. It is also still controversial whether low $\mathrm{O}_{2}$ concentrations can be adopted as the standard for human embryo culture, at least for blastocyst formation. Thus, one of aims in our current study is to find out the relationship between $\mathrm{O}_{2}$ concentration and embryo hatching ability in vitro.

Furthermore, many oxygen-regulated genes have been proved to function in embryonic development and metabolish [9, 28], whereas it remains poorly understood whether there are any oxygen-regulated imprinted genes, connecting with the process of blastocyst hatch. Parthenogenetic embryos produced in vitro enable us to study the effect of maternally expressed genes in preimplantation embryos, since it is still unknown how parental genetic background influence embryo hatching [29, 30].

Besides that, sperm's penetration through the ZP during fertilization, triggers the exocytosis of cortical granules in oocytes and biochemically alters the structure of ZP, thus making it impermeable to additional sperm, to prevent polyspermy [31-34]. So the integrity of ZP structure in normally in vivo fertilized embryos, is distinctly different from that in parthenogenetic embryos activated by chemicals in vitro, which is intact and completely hermetical. This provides us a powerful tool to study the effects of physical structural changes of ZP on embryo hatching process.

Therefore, this study aims to determine the effects of oxygen concentrations, parental genetic material, and integrity of ZP structure on blastocyst hatch, and tries to find out the molecular mechanisms regulating the blastocyst hatch, by utilizing fertilizated and parthenogenetic mouse embryos collected and cultured in vitro.

\section{Methods}

Unless otherwise specified, all reagents were obtained from Sigma Chemical Co. (St. Louis, MO).

\section{Animal care and ethics statement}

B6C3F1 mice were maintained in an environmentally controlled room at $22 \pm 1{ }^{\circ} \mathrm{C}$, under a light cycle of $14 \mathrm{~h}$ light/10 $\mathrm{h}$ dark, with free access to food and water in animal facility of specific pathogen-free (SPF) in Sun Yat-sen University. All experimental protocols involving the handling of mice were approved by the Institutional Animal Care and Use Committee of Sun Yat-sen University (Approval No. IACUC-2014-0102), People's Republic of China.

\section{Collection of mouse oocytes and zygotes}

Collection and culture of zygotes were performed as previously described [35-37]. B6C3F1 female mice aged at 5-8 weeks (weighed 16-19 g) were superovulated with 5 IU pregnant mare serum gonadotropin by intraperitoneal injection (PMSG, Calbiochem, La Jolla, CA), followed by 5 IU human chorionic gonadotrophin (hCG) 46-48 h later. Female mice were mated individually with B6C3F1 males of proven fertility, and successfully mated females indicated by a vaginal plug in the next morning, were used for collecting zygotes (day 0.5) at 20-21 h after hCG injection. Oocytes enclosed in cumulus masses were collected from oviduct ampullae $14 \mathrm{~h}$ after hCG injection. Cumulus cells were removed by pipetting after brief incubation in $0.03 \%$ hyaluronidase prepared in potassium simplex optimized medium (KSOM) containing $14 \mathrm{mM}$ Hepes and $4 \mathrm{mM}$ sodium bicarbonate (HKSOM). After removal of cumulus cells, zygotes and oocytes were then washed and incubated in $50 \mu \mathrm{L}$ droplets of pre-equilibrated KSOM supplemented with nonessential and essential amino acids and $2.5 \mathrm{mM}$ hepes $\left(\mathrm{KSOM}_{\mathrm{AA}}\right)$ and then covered with mineral oil pending further treatments. Zygotes and Oocytes culture and all subsequent embryonic culture experiments were carried out at $37{ }^{\circ} \mathrm{C}$ in a humidified atmosphere of $6.0 \% \mathrm{CO}_{2}$ in air. All manipulations in vitro were performed at $36-37^{\circ} \mathrm{C}$ on heated stages or in incubators. Embryos normally cleaved to two-cells at day 1-1.5, and formed morulae at 
day 2.5 (48 $\mathrm{h}$ in culture). Early blastocysts were formed at day 3.5 (72 $\mathrm{h}$ in culture), and late expanded blastocysts at day 4.5 (96 h in culture). Hatched blastocysts appeared at day5.5 (120 $\mathrm{h}$ in culture).

\section{Parthenogenetic activation of oocytes}

Freshly ovulated metaphase II oocytes were collected from the ampullae $14 \mathrm{~h}$ after hCG injection, and cumulus cells were removed as described above. Diploid parthenogenetic embryos were produced by activation of oocytes with $\mathrm{SrCl}_{2}$ and cytochalasin D in $20 \% \mathrm{O}_{2}$, as described before [30].

\section{Embryo culture in vitro}

Both zygotes and parthenogenetic embryos were cultured in groups of 20-30 embryos per $50 \mu \mathrm{L}$ droplet of $\mathrm{KSOM}_{\mathrm{AA}}$ overlaid with embryo-tested mineral oil. Embryos were cultured in humidified atmospheres (Thermo) with $5 \%$ or $20 \% \mathrm{O}_{2}$. Culture plates (35-mm petri dish; Corning Inc., Corning, NY) were prepared and equilibrated in the incubator $1 \mathrm{~h}$ before embryos collection. All control groups were made in the same day and the same treatment groups were cultured in the same dish with distinct makers. In all experiments, embryos were cultured for 5 days in vitro, and observed respectively at 24, 72, 96 and $120 \mathrm{~h}$ in vitro and graded for the stage of development including blastula formation and hatch.

\section{Embryo hypotonicity}

The embryos culured for $48 \mathrm{~h}$ were transferred into a $20 \mu \mathrm{L}$ droplet of pure water under mineral oil in $35 \mathrm{~mm}$ Petri dish directly, and immediately observed under Zeiss inverted microscope for live image.

\section{Zona pellucida drilling by Peizo micromanipulation}

Ovulated metaphase II oocytes were collected at $14 \mathrm{~h}$ after hCG, and transferred into HKSOM microwell. A tiny breach (about $1 \mu \mathrm{m}$ ) on ZP was drilled with a pronuclear injection pipette or a $5 \mu \mathrm{m}$ blunt-end pipette by Piezo micromanipulator.

\section{Live image with embryo}

Each 4 un-hatched blastocysts cultured for nearly 96 or $120 \mathrm{~h}$ in vitro were transferred into $2 \mu \mathrm{L}$ HKSOM medium covered with mineral oil in a $35 \mathrm{~mm}$ coated glass bottom microwell dish (MatTek corporation). The dish was put on a heating plate controlled by a temperature controller (Medical systems corp. Model: TC-202A) to keep $37{ }^{\circ} \mathrm{C}$. Zeiss inverted microscope and AxionVision LE software were used to make live image.

\section{Immunofluorescence (IF) staining}

Embryos were washed twice in phosphate buffered saline containing $0.1 \%$ polyvinyl pyrrolidone (PBS-PVP), then fixed in freshly prepared $3.7 \%$ paraformaldehyde in PBSPVP ( $\mathrm{pH} 7.4$ ) for $15 \mathrm{~min}$ at $4{ }^{\circ} \mathrm{C}$, permeablized in $0.1 \%$ Triton X-100 in blocking solution (3\% goat serum in PBS-PVP) for $30 \mathrm{~min}$, washed three times, and then left in blocking solution for $1 \mathrm{~h}$. Embryos were incubated at $4{ }^{\circ} \mathrm{C}$ overnight with anti-Oct4 mouse monoclonal antibody (sc5279, santa cruz) diluted 1:50 in blocking solution, washed, and then incubated for $1 \mathrm{~h}$ with secondary antibodies conjugated to Alexa Fluor 568 (Molecular Probes) diluted 1:100 in blocking solution. Embryos were washed, and mounted onto a slide under a coverslip in the Vectashield with $0.2 \mu \mathrm{g} / \mathrm{mL}$ Hoechst 33342 mounting medium. Alexa Fluor-labeled Oct4 and Hoechst-labeled nuclei were observed with a Leica inverted fluorescence microscope.

\section{Detection of apoptosis by TUNEL assay}

Embryos were collected, washed three times in PBSPVP, and fixed in 3.7\% paraformaldehyde overnight at $4{ }^{\circ} \mathrm{C}$. Nuclear DNA fragmentation was detected by the TUNEL method using the In Situ Cell Death Detection Kit (Fluorescein, Roche Applied Science) according to the manufacturer's instructions, and nuclei were counterstained with propidium iodide (PI, $50 \mu \mathrm{g} / \mathrm{mL}$, Sigma). Fluorescence was detected using a Leica inverted fluorescence microscope.

\section{Real-time PCR relative quantitation of gene expression}

Thirty blastocysts from both fertilized and parthenogenetic groups cultured in $5 \%$ or $20 \% \mathrm{O}_{2}$ for $96 \mathrm{~h}$ in vitro were collected and used for mRNA extraction with RNeasy micro Kit (Qiagen, 74004). Total mRNA was subjected to cDNA synthesis using Reverse Transcription System (Toyobo). Primers for amplification of all the selected genes were designed using GeneTool Lite 1. 0 (BioTools) (see Additional file 1). Polymerase chain reaction (PCR) was performed with ABI Prism 7900 Sequence Detection System (Applied Biosystems) using SYBR Green Real-time PCR Master Mix (Toyobo, QPK-201). Gene expression levels were analyzed and normalized to housekeeping gene $\beta$-actin as the reference gene.

\section{Statistical analysis}

Percentages were transformed using arcsin transformation. Unless otherwise indicated, percentage transformed data, cell number, embryo hatch time and real-time PCR data were analyzed by one-way analysis of variance (ANOVA) and means were compared by Fisher's protected least-significant difference (PLSD) using the StatView software from SAS Institute Inc. (Cary, NC). Significant difference was defined as $P<0.05$. 


\section{Results}

Both the oxygen concentration and genetic material affect the blastocyst hatch process in vitro

To assess the effects of oxygen concentration and genetic material on embryo development and hatch process, we carried out four combinatorial treatments in a twofactor design by using the in vitro model of embryo culture, in which both fertilized zygotes from naturally mated female mice and parthenogenetic embryos from chemically activated MII oocytes were cultured for $120 \mathrm{~h}$ in 5 and $20 \% \mathrm{O}_{2}$ respectively.

No detectable differences were observed in the blastocyst rates (around 97\%) and morphology, among these four groups cultured for $96 \mathrm{~h}$ (Table 1 and Fig. 1a). However, the hatch rates among these groups showed significant differences after culture for $120 \mathrm{~h}$ (Table 1 and Fig. 1b). About 58.2\% of fertilized embryos in $20 \% \mathrm{O}_{2}$ hatched, which was notably higher than those in $5 \% \mathrm{O}_{2}$ (23.8\%, Table 1 and Fig. 1b). Conversely, the percentage of hatched parthenogenetic blastocysts in $5 \% \mathrm{O}_{2}(90.8 \%)$ was significantly increased, compared with that in $20 \% \mathrm{O}_{2}(81.2 \%$, Table 1 and Fig. 1b). These results demonstrate that the oxygen concentration does not affect the development of either fertilized or parthenogenetic embryos to blastocyst (day 3), but influences the blastocyst hatch following blastulation (days 5). Interestingly, $5 \% \mathrm{O}_{2}$ reduced the hatch ratio of fertilized embryos, but increased that in parthenogentic embryos, suggesting that there are distinct mechanistic differences between fertilized and parthenogentic embryos in regulating the effects of oxygen on blastocyst hatch. Moreover, the hatch ratio of parthenogenetic embryos was markedly higher than that of fertilized embryos, regardless of oxygen concentration (Table 1), implying a significant influence of parental genetic background on blastocyst hatch.

\section{The allocation of ICM cells is affected diversely in} fertilized and parthenogenetic blastocysts in vitro

To better understand the mechanism regulating embryo hatch, immunostaining for Oct4 protein, a crucial marker for ICM and embryonic stem cells (ESCs), and TUNEL assay were performed to check the cell allocation, apoptosis, and total embryonic cell number in these blastocysts among groups after culture for $120 \mathrm{~h}$. There were no overt differences for the number of Oct4-positive cells in either fertilized or parthegenetic groups, when embryos cultured in $20 \% \mathrm{O}_{2}$ was compared with those in 5\% (Table 2 and Fig. 2a). Likewise, we found no any change for the number of apoptotic cells among groups, with less than two apoptotic cells observed in all groups of blastocysts by TUNEL assay (Table 2 and Fig. 2b). Congruently, when they were cultured for $96 \mathrm{~h}$, that is, prior to hatching, embryos among groups had a comparable mRNA expression level of both Oct4 and apoptosis-associated genes, $B c l 2$ and Bax, confirmed by real-time PCR analysis (Fig. 2c).

However, the ratio of Oct4-positive cells in each embryo after culture for $120 \mathrm{~h}$ varied considerably among these groups, due to a marked difference in the total embryonic cell number (Table 2). When compared with $20 \% \mathrm{O}_{2}$, obviously, $5 \% \mathrm{O}_{2}$ decreased the ratio of Oct4positive cells in fertilized blastocysts, whereas increased that in pharthenogenetic blastocysts. This result resembles the data on hatch ratio (Table 1), and thus suggests a possible regulatory role for the allocation of ICM cells in blastocyst hatch. Furthermore, we found that the total cell number of fertilized embryos was higher than that of parthegenetic embryos in both 5\% and 20\% $\mathrm{O}_{2}$ (Table 2), and that $5 \% \mathrm{O}_{2}$ increased the total cell number in both fertilized and parthenogenetic embryos, comparing to $20 \% \mathrm{O}_{2}$ (Table 2). These data unambiguously illustrates the importance of both the parental genetic material and oxygen concentration on preimplantation development, and thus subsequent embryo hatch.

\section{Oxygen concentration affects the gene expression in fertilized and parthenogenetic blastocysts before hatching}

To find the key genes involved in the control of embryo hatch, several important genes involving in different cellular events were analyzed by real-time PCR, using fertilized and parthenogenetic blastocysts cultured in vitro for $96 \mathrm{~h}$ under $5 \%$ or $20 \% \mathrm{O}_{2}$. Firstly, the expression patterns of three common imprinted genes in these

Table 1 Development potential of fertilized and parthenogenetic embryos cultured in 5 and 20\% oxygen in vitro

\begin{tabular}{llllll}
\hline Groups & $\begin{array}{l}\text { Oxygen } \\
\text { concentration \% }\end{array}$ & Replicates No. & $\begin{array}{l}\text { Total } \\
\text { embryos No. }\end{array}$ & $\begin{array}{l}\text { No. (\%) } \\
\text { Blastocysts }\end{array}$ & $\begin{array}{l}\text { ( } \\
\text { No. (\%) } \\
\text { Hatched Blastocysts }\end{array}$ \\
\hline FE & 20 & 7 & 324 & $312(96.2 \pm 1.4)$ & $179(58.2 \pm 4.2)^{\mathrm{a}}$ \\
& 5 & 7 & 350 & $337(97.3 \pm 1.1)$ & $83(23.8 \pm 2.6)^{b}$ \\
PA & 20 & 9 & 346 & $336(97.6 \pm 0.8)$ & $278(81.2 \pm 2.7)^{\mathrm{c}}$ \\
& 5 & 9 & 346 & $335(96.8 \pm 1.0)$ & $306(90.8 \pm 1.8)^{\mathrm{d}}$ \\
\hline
\end{tabular}

FE fertilized embryos, $P A$ parthenogenetically activated embryos

${ }^{+}$Mean \pm SEM

${ }^{a}$ vs. ${ }^{b}, p<0.001 ;{ }^{a}$ vs. ${ }^{c}, p<0.01 ;^{c}$ vs. ${ }^{d}, p<0.05 ;{ }^{b}$ vs. ${ }^{d}, p<0.001$ 


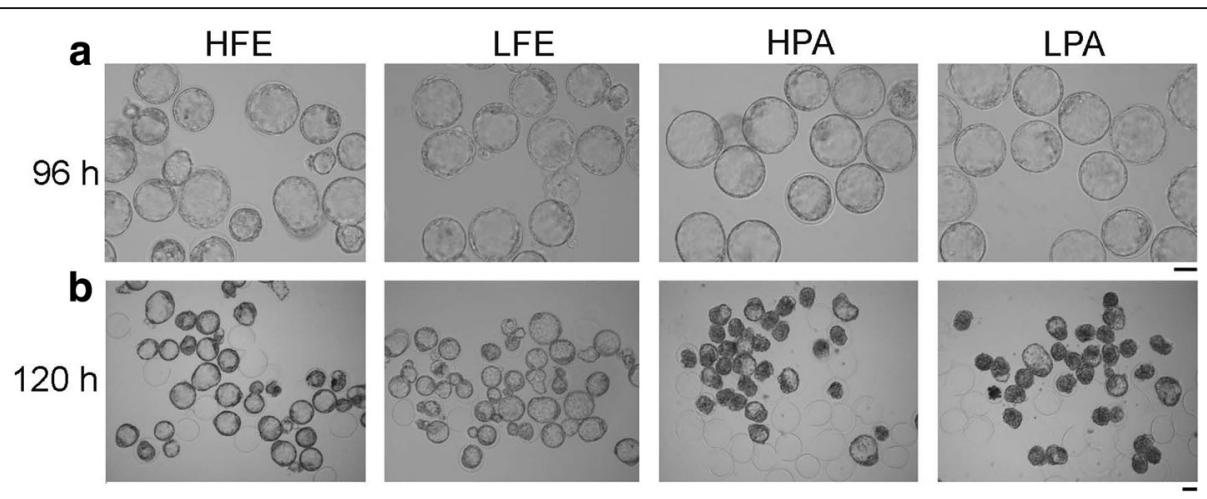

Fig. 1 Morphology and hatch process of embryos cultured in vitro. a Morphology of fertilized and parthenogenetic embryos cultured in vitro for $96 \mathrm{~h}$ in $20 \%$ or $5 \% \mathrm{O}_{2}$. Bar $=50 \mu \mathrm{m}$. b Morphology of fertilized and parthenogenetic embryos cultured in vitro for $120 \mathrm{~h}$ in $20 \% \mathrm{or} 5 \% \mathrm{O}_{2}$. Bar $=50 \mu \mathrm{m}$. HFE and LFE, fertilized embryos cultured in 20\% and 5\% $\mathrm{O}_{2}$ respectively; HPA and LPA, parthenogenetic embryos cultured in 20\% and 5\% $\mathrm{O}_{2}$ respectively

four groups differed greatly as following: the expression of maternally-expressed $H 19$ was significantly increased but paternally-expressed Snrpn notablely reduced in both parthenogenetic embryo groups, when compared with fertilized embryo groups, and the expression of Igf $2 r$ was not changed among groups (Fig. 3a), indicating that their expressions are influenced only by the parental genetic background, rather than oxygen concentration. Secondly, the proteinase genes, Pitrm1, Prnt3, Psmc4 and Psmd11, which were reported to function in regulating the hatch process $[7,38]$, demonstrated a similar expression level in all groups (Fig. 3b). Given that the mitogen-activated protein kinase (MAPK) signal pathway plays a key role in embryonic cell division [39], then, the expressions of three important regulating genes in this pathway were confirmed including Erk1, Erk2 and Gab1, since we have found a significant change in the total cell number among groups (Table 2). As expected, the expression of all three genes in fertilized embryo groups were significantly increased in $5 \% \mathrm{O}_{2}$ group (Fig. 3c). Similarly, in parthenogenetic embryo groups, Erk1 expression significantly rised and Erk2 and Gab1 slightly increased in $5 \% \mathrm{O}_{2}$ group (Fig. 3c). What's more, Gab1 expression was significantly reduced in parthenogenetic embryos in both 5 and $20 \% \mathrm{O}_{2}$, when compared to paralleled fertilized embryos (Fig. 3c), showing a expression pattern similar to imprinted gene Snrpn. Finally, we checked the expression of $\operatorname{Sod} 1$ and $\operatorname{Sod} 2$, two menbers from superoxide dismutases (SODs) family, which is the first and most important line of antioxidant enzyme defense systems against reactive oxygen species (ROS) and can thus help embryo developing well in vitro $[40,41]$. While there was no difference found among all groups for $\operatorname{Sod} 2$ expression, slightly higher expression level of Sod1 was observed in parhtenogenetic embryos, when compared with fertilitzed embryos (Fig. 3d). There results clearly illuminate that oxygen concentration affects the gene expression in fertilized and parthenogenetic embryos, suggesting a potential role of MAPK in regulation of the preimplantation development and consequent blastocyst hatch in vitro.

\section{Sperm-drilling breach is not the main reason for different hatch dynamics between fertilized and parthenogenetic embryos}

To furthur elucidate how the embryo hatch was affected in both fertilized and parthenogenetic embryos, we monitored the embryo hatch process by living imaging and found that fertilized embryos spend about $42 \mathrm{~h}$ completing the hatch process, while parthenogenetic embryos spend only $6 \mathrm{~h}$ (see Additional files 2 and 3, Table 3). In detail, we found that fertilized embryos started hatching

Table 2 Cell allocation and apoptosis in fertilized and parthenogenetic embryo cultured in vitro for $120 \mathrm{~h}$

\begin{tabular}{|c|c|c|c|c|c|c|}
\hline \multirow[t]{2}{*}{ Groups } & \multirow[t]{2}{*}{ No. embryos } & \multicolumn{3}{|c|}{120 h Blastocysts $^{+}$} & \multirow[t]{2}{*}{ No. embryos } & \multirow{2}{*}{$\begin{array}{l}120 \text { h Blastocysts } \\
\text { No. apoptosis cells }\end{array}$} \\
\hline & & No. total cells & No. Oct4 positive cells & $\begin{array}{l}\text { Oct4 positive cells/ } \\
\text { Total cells (\%) }\end{array}$ & & \\
\hline HFE & 37 & $116.0 \pm 3.2^{a}$ & $25.2 \pm 1.2$ & $21.7 \pm 0.8^{e}$ & 14 & $1.9 \pm 0.6$ \\
\hline LFE & 39 & $129.4 \pm 4.4^{b}$ & $24.5 \pm 1.2$ & $18.8 \pm 0.6^{f}$ & 13 & $1.2 \pm 0.6$ \\
\hline HPA & 49 & $105.7 \pm 2.6^{c}$ & $23.2 \pm 1.0$ & $21.9 \pm 0.7^{e}$ & 11 & $1.4 \pm 0.4$ \\
\hline LPA & 60 & $112.6 \pm 2.7^{\mathrm{acd}}$ & $27.6 \pm 0.9$ & $24.7 \pm 0.6^{9}$ & 12 & $1.3 \pm 0.4$ \\
\hline
\end{tabular}

HFE and LFE fertilized embryos cultured in 20 and 5\% oxygen, HPA and LPA parthenogentic embryos cultured in 20 and $5 \%$ oxygen

${ }^{+}$Mean \pm SEM

${ }^{a}$ vs. ${ }^{b}, p<0.01 ;{ }^{a}$ vs. ${ }^{c}, p<0.05 ;{ }^{b}$ vs. ${ }^{c}, p<0.0001 ;{ }^{b}$ vs. ${ }^{d}, p<0.001 ;{ }^{c}$ vs. ${ }^{d}, p<0.05 ;{ }^{e}$ vs. ${ }^{f},{ }^{g} p<0.01 ;{ }^{f}$ vs. ${ }^{g}, p<0.01$ 

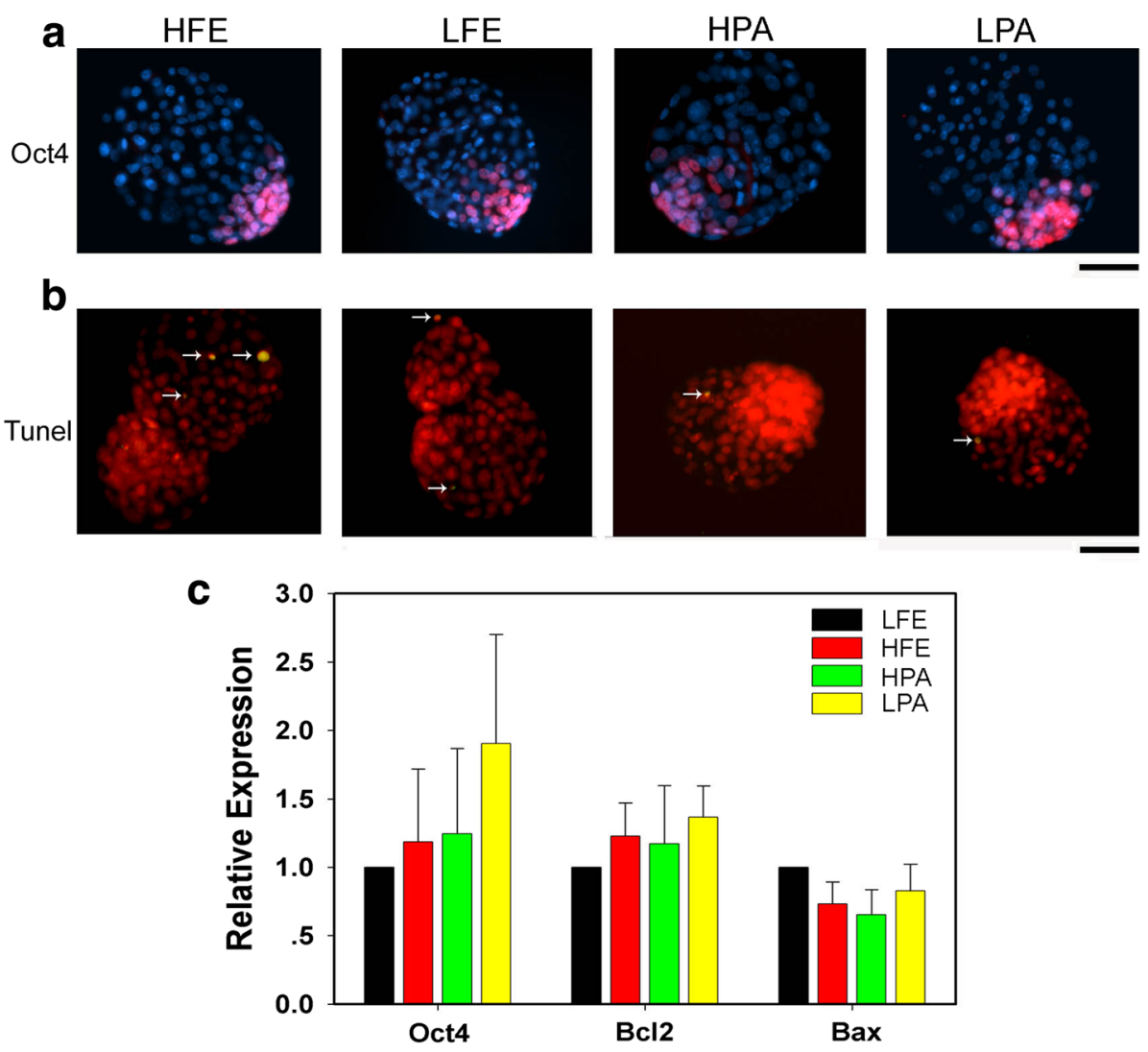

Fig. 2 Embryonic cell differentiation and apoptosis in blastocysts cultured in vitro. a Immunofluorescent images showing Oct4-positive cells in fertilized and parthenogenetic blastocysts cultured for $120 \mathrm{~h}$ in 5\% or 20\%, indicative of inner cell mass (pink), with nuclei stained with Hoechst (blue). Bar $=50 \mu \mathrm{m}$. b Representative merged images showing apoptotic cells (yellow, white arrows) in fertilized and parthenogenetic blastocysts cultured for $120 \mathrm{~h}$ in $5 \%$ or $20 \%$ by TUNEL assay, with nuclei stained with PI (red). Bar $=50 \mu$ m. c Quantification of Oct4 and apoptotic genes, including $\mathrm{BCl} 2$ and $\mathrm{Bax}$, in fertilized and parthenogenetic embryos cultured for $96 \mathrm{~h}$. Relative mRNA expression levels are determined by real-time PCR and normalized to $\beta$-actin as internal reference, with the average value of LFE groups as control set to one and compared across groups. Data are presented as mean \pm SEM. LFE and HFE, fertilized embryos cultured in 5\% and 20\% $\mathrm{O}_{2}$ respectively; LPA and HPA, parthenogenetic embryos cultured in $5 \%$ and $20 \% \mathrm{O}_{2}$ respectively

at $72 \mathrm{~h}$ and about $43 \%$ completed hatching at $120 \mathrm{~h}$ in $20 \% \mathrm{O}_{2}$ (Fig. $4 \mathrm{a}$ and Table 3). Nevertheless, none of the $71 \%$ parthonegenetic embryos started to hatch until $120 \mathrm{~h}$, without any signs observed at 72 or even at $96 \mathrm{~h}$ (Fig. 4a and Table 3), indicating a significant difference in the hatch dynamics between fertilized blastocysts and parthonegenetic blastocysts.

Since sperm penetration through the oocyte ZP makes the resultant fertilized embryos different from parthenogenetic embryos in the physical structure of $\mathrm{ZP}$, we wondered whether this divergence could also lead to marked differences in hatch process. To answer this question, we performed the hypotonic experiment using embryos cultured for $48 \mathrm{~h}$. Significantly, the cytoplasm permeated out through a breach on ZP from all fertilized embryos after transferred into pure water, while no cytoplasm leaked out in parthenogenetic embryos (Fig. 4b, Additional files 4 and 5), indicating that the breach generating from sperm's penetration may be the reason leading to two different hatched mechanisms.

In order to verify this possiblibity, a tiny breach (about $1 \mu \mathrm{m})$ was artificially made on the mature oocyte ZP using pronuclear injection method. After the oocytes were parthenogenetically activated and then cultured for $48 \mathrm{~h}$, we found that no cytoplasm permeated out from the ZP-drilled parthenogenetic embryos in hypotonic experiment, and no hatching embryo was observed before $96 \mathrm{~h}$, and 80\% embryos (8/10, Table 3$)$ hatched in $120 \mathrm{~h}$, indicating the same hatch pattern as normal parthenogenetic embryos. One of the potential reasons for the above results is that the breach drilled by pronuclear injection pipette was much smaller than that generated by sperm penetration in fertilized embryo, which would block the cytoplasm outflowing in hypotonic experiment. Thus, a wider injection pipette (approximate $5 \mu \mathrm{m}$ in blunt-end) was used to make a larger breach on 

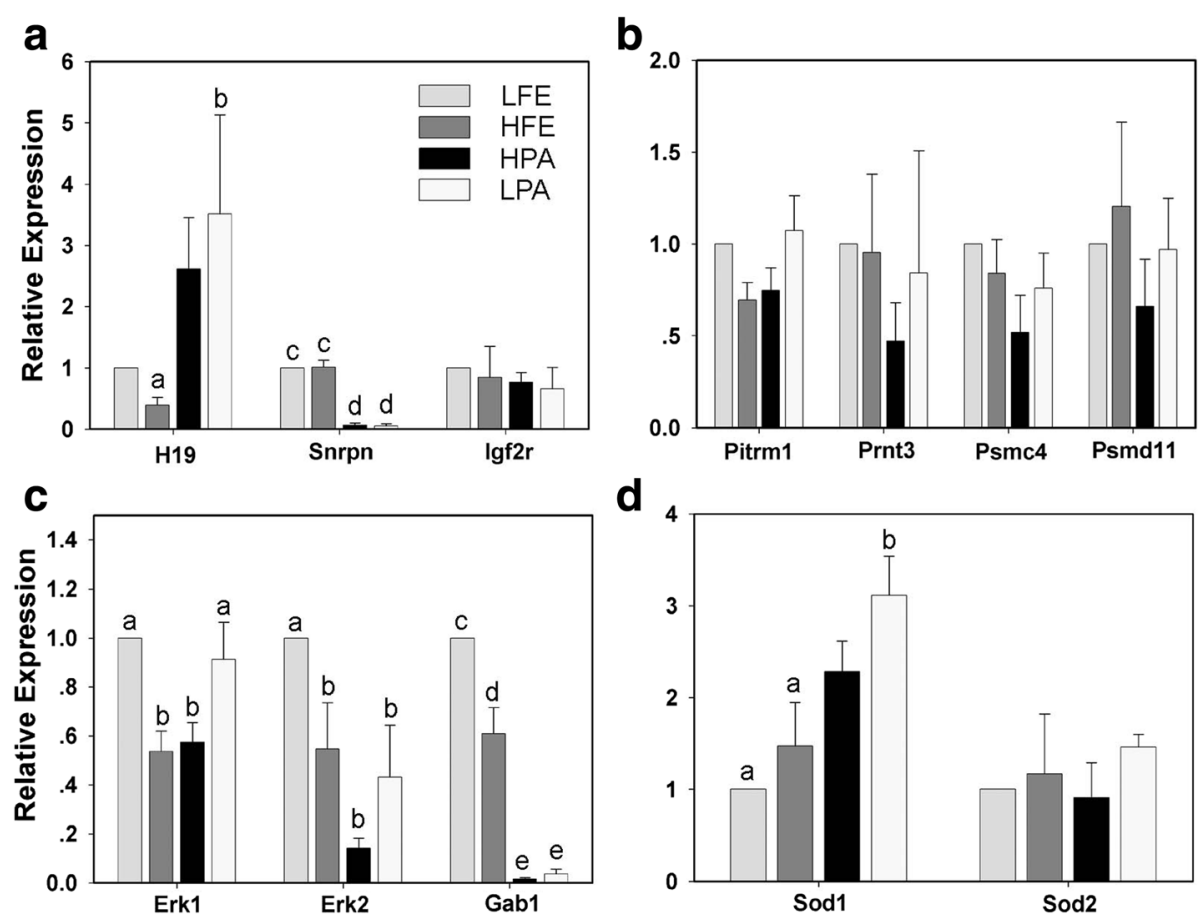

Fig. 3 Quantification of key genes expression in embryos cultured in vitro for $96 \mathrm{~h}$. a Imprinted genes expression. b Proteinase genes expression. c MAPK signal pathway genes expression. d Superoxide dismutase genes expression. LFE and HFE, fertilized embryos cultured in $5 \%$ and $20 \% \mathrm{O}_{2}$ respectively; LPA and HPA, parthenogenetic embryos cultured in $5 \%$ and $20 \% \mathrm{O}_{2}$ respectively. Gene-expression data are determined by real-time PCR and normalized to the levels of $\beta$-actin mRNA, with the average value of LFE groups as controls set to one and compared across groups. Data are presented as mean \pm SEM, with letters indicating differences between groups, a vs. $b, p<0.05$; c vs. $d, p<0.001$; c vs. e, $p<0.0001$; d vs. e, $p<0.0001$

oocyte ZP at similar size as sperm penetration in fertilized embryos. After hypotonic treatment at $48 \mathrm{~h}$, the cytoplasm permeated out from ZP-drilled parthenogenetic embryos (Fig. 4b, Additional file 6). Furthermore, ZP-drilled parthenogenetic embryos started to hatch at $96 \mathrm{~h}$, and $75 \%$ embryos $(15 / 20)$ completed hatching in $120 \mathrm{~h}$, which were similar to normal parthenogenetic embryos but significantly higher than fertilized embryos (Fig. 4a and Table 3). However, we found that the blastocysts did not come out from the aritificial breach on ZP, but kept expanding and then hatched out from another place of ZP suddenly and quickly, although some trophoderm cells had already came out through the spermlike breach at $96 \mathrm{~h}$ (see Additional file 7). These results indicate that the sperm-like breach on ZP was not the main reason for the mechanistic difference of hatch between parthenogenetic and fertilized embryos.

\section{Discussion}

Uterus interacts with embryos and secretes lots of lytic factors to help embryo hatch and implant [42, 43]. Whereas, uterus is not the determinant issue for embryo hatch, since the embyos are able to hatch by themselves in vitro, through a way different from that developed in vivo $[13,44]$. Assisted hatching $(\mathrm{AH})$, an invasive technique aiming to facilitate embryo implantation by artificially manipulating zona pellucida in vitro, has been widely used, although its clinical efficacy remains controversial [4, 45-47]. Understanding molecular mechanisms involving in embryo hatching will enable the

Table 3 Hatched percentage and time of different types of embryo

\begin{tabular}{llllll}
\hline Embryo types & $\begin{array}{l}\text { Diameter of hole } \\
\text { on ZP }\end{array}$ & No. embryos & 96 h Blastocysts & $\begin{array}{l}120 \text { h Hatched } \\
\text { Blastocysts (\%) }\end{array}$ & $\begin{array}{l}\text { Total Hatched } \\
\text { time (repeats) }\end{array}$ \\
\hline FE & Sperm-size & 23 & 23 & $10(43)^{\mathrm{a}}$ & $41.7 \pm 5.1^{\mathrm{c}}(9)$ \\
PA & None & 24 & 24 & $17(71)^{\mathrm{b}}$ & $6.0 \pm 1.8^{\mathrm{d}}(10)$ \\
PA-ZPI & $\sim 1 \mu \mathrm{m}$ & 10 & 10 & $8(80)^{\mathrm{b}}$ & $15(75)^{\mathrm{b}}$ \\
PA-ZPI & $\sim 5 \mu \mathrm{m}$ & 20 & 20 &
\end{tabular}

${ }^{\mathrm{a}}$ vs. ${ }^{\mathrm{b}}, p<0.05 ;{ }^{\mathrm{c}}$ vs. ${ }^{\mathrm{d}}, p<0.0001$ 


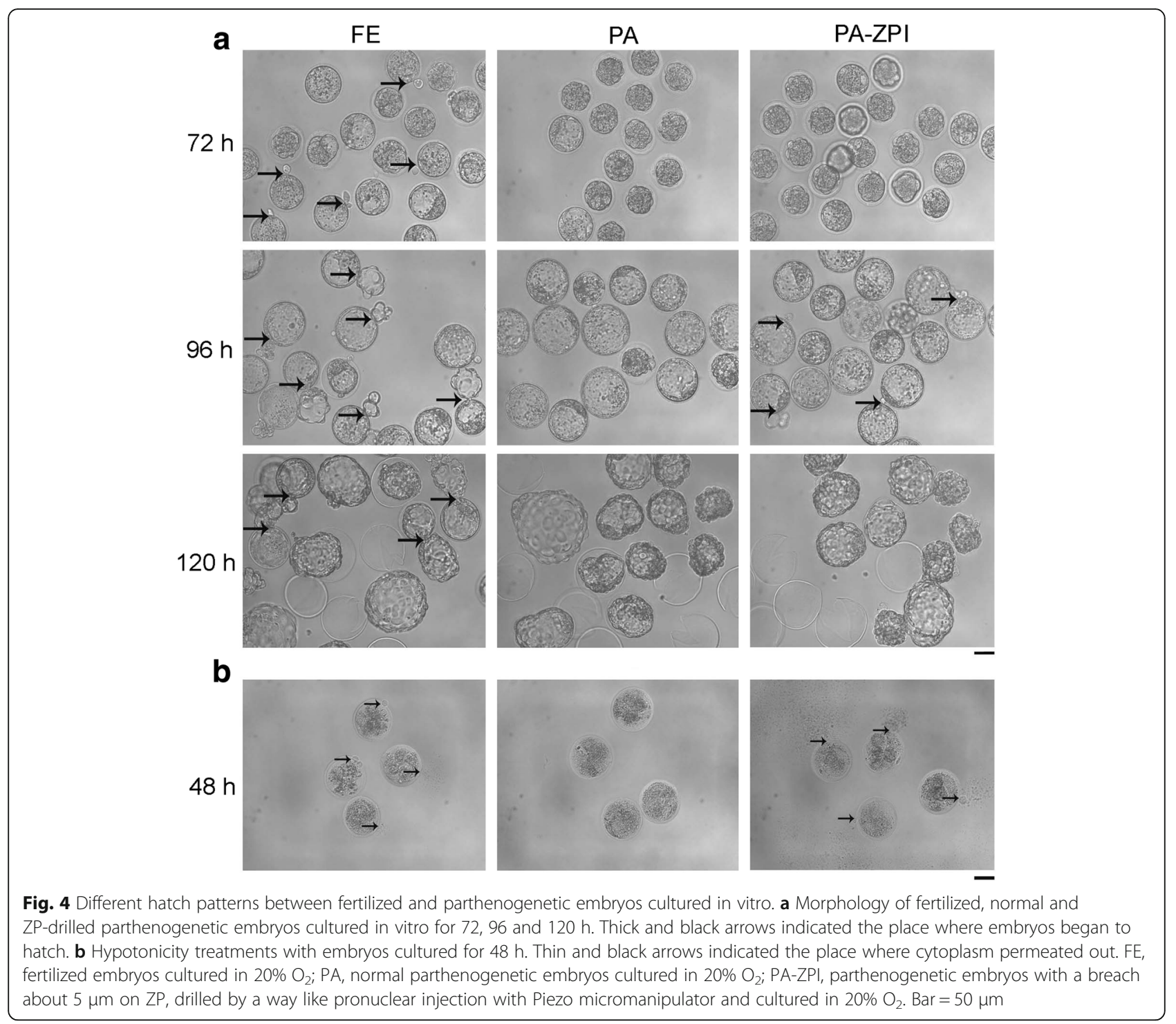

development of new interventions for embryo implantation and thus improve the human reproductive outcomes. Here, our results indicate that the mechanism of mouse embryo hatch is regulated by both intrinsic and extrinsic factors in vitro.

\section{Both oxygen concentration and parental genetic material affect preimplantation development and subsequent blastocyst hatching}

Oxygen concentration is one of the most important extrinsic factors for embryo culture in vitro [15-17]. In our present study, we found that both fertilized and parthenogenetic embryos developed to blastocyst stage well in $5 \%$ and $20 \% \mathrm{O}_{2}$ at $96 \mathrm{~h}$, which means that oxygen concentration does not affect embryo developmental morphology, as reported in bovine [17], mouse [19], and human $[26,27]$. However, after culture for $120 \mathrm{~h}$, that is, after blastocyst formation, we found that $5 \% \mathrm{O}_{2}$ increased the total cell number in both fertilized and parthenogenetic embryos, comparing to $20 \% \mathrm{O}_{2}$. This result is accordant with a previous conclusion that $5 \% \mathrm{O}_{2}$ is better for mouse embryo development [19, 22, 48, 49]. Moreover, in both $5 \%$ and $20 \% \mathrm{O}_{2}$, the total cell number of fertilized embryos was significantly higher than that of parthegenetic embryos. These data indicate that both oxygen concentration and parental genetic background can regulate embryonic cell proliferation after blastulation, and thus affect the subsequent hatch process in vitro.

As for the effects of oxygen concentration on hatch, we found that $5 \% \mathrm{O}_{2}$ reduced the hatch rate of fertilized embryos, which is consistent with Fischer's report that bovine fertilized embryos have notablely higher hatched rate cultured under $20 \% \mathrm{O}_{2}$ in KSOM medium [17]. On 
the contrary, we found that $5 \% \mathrm{O}_{2}$ increased the hatch rate in parthenogentic embryos, which suggests that the mechanism regulating the effects of oxygen concentration on hatch process in parthenogentic embryos is distinctly different from that in fertilized embryos. Moreover, the hatch ratio of blastocysts showed significant differences among groups after culture for $120 \mathrm{~h}$, and implied a significant effect of maternal genetic background on embryo hatch, revealed by the markedly higher hatch ratio observed in parthenogenetic embryos than fertilized embryos, regardless of oxygen concentration.

We suppose that $5 \% \mathrm{O}_{2}$ increased the total embryonic cell number in both fertilized and parthenogenetic blastocysts, mainly by stimulating TE cells proliferation, since we did not find any difference in either embryo qualities, indicated by the similar number of apoptotic cells and camparable expression level of apoptosis related genes in all embryo groups, or embryonic cell differentiation, indicated by no changes for the expression level of pluripotent gene Oct4 and the number of Oct4positive cells that are considered as ICM cells [50]. Nevertheless, comparing with $20 \% \mathrm{O}_{2}$, the reason why $5 \% \mathrm{O}_{2}$ inhibit fertilized embryos but promote pharthenogenetic embryos hatching remains unclear. In this study, we amazedly found that $5 \% \mathrm{O}_{2}$ significantly decreased the ratio of Oct4-positive cells in fertilized blastocysts, whereas increased that in pharthenogenetic blastocysts. That is to say, both fertilized and parthenogenetic embryos with higher hatched rate, have higher ratio of Oct4-positive cells accordingly, thus indicating a potential close relationship between the allocation of ICM cells and subsequent blastocyst hatch. We speculate that embryos with higher ICM ratio likely secrete more factors, which may be benefiticial for embryo hatch [42, 43].

Taking into account that oxygen can regulate the expression of embryonic genes, and that low oxygen can affect the expression of oxygen-regulated genes in mouse and bovine embryos $[9,16,28]$, we performed real-time PCR to analyze the expression levels of key genes that may be involved in the control of embryo hatch, by using fertilized and parthenogenetic blastocysts cultured in $5 \%$ and $20 \% \mathrm{O}_{2}$ for $96 \mathrm{~h}$, when the mRNA transcript expression level would be changed at this stage, if any of these genes is functioned in regulating embryo hatch. Since we found that the expression level of three common imprinted genes including H19, Snrpn, and Igfr2, are influenced only by the parental genetic background, as previously reported [16], further studies are required to better characterize whether there are any aother imprinted genes involving in the blastocyst hatch process. Also, our results showed no changes for all the four proteinase genes including Pitrm1, Prtn3 and Psmd11 as well as Psmc, indicating that these proteinase genes might not be impacted by oxygen concentration during embryo hatching, although they were demonstrated to be important for embrygenesis and embryo hatching in previous studies [7, 38].

However, we found that $5 \% \mathrm{O}_{2}$ significantly indeed increased Erk1 and Erk2 expression in both fertilized and parthenogenetic embryos, which is one of the reasons why $5 \% \mathrm{O}_{2}$ can promote embryonic cell proliferation, indicated by increased total embryonic cell number, when compared with $20 \% \mathrm{O}_{2}$. Simultaneously, the expression level of Gab1, another gene in MAPK pathway, was significantly increased in fertilized embryos in both $5 \%$ and $20 \% \mathrm{O}_{2}$, which accounts for their significantly higher total cell number, when compared to paralleled parthegenetic embryos. Furthermore, we analyzed the expression level of two genes from superoxide dismutases (SODs) family, and found no change for Sod2 expression, whereas slight higher expression level of Sod1 was observed in parhtenogenetic embryos, when compared with fertilitzed embryos. Given that superoxide dismutases can protect against reactive oxygen species (ROS) $[40,41]$, we hence presume that reduced oxidative stress, due to increased sod1 expression, may be one of the mechanisms to faciliate embryo hatch, indicated by the higher hatch rate in parhtenogenetic embryos. But, this hypothesis might not be suitable for normal fertilized embryos, because of the distinct differences in mechanisms regulating the hatch process between fertilized and parthonegenetic blastocysts. Therefore, it will be meaningful to further explore whether antioxidants, such as N-Acety-L-Cysteine (NAC) or resveratrol $[37,51]$, can increase the hatch rate of human embryos and thereby improve the clinic outcomes, since the relationship between the oxidative stress and embryo hatch remains largely unknown.

\section{Sperm-like breach induces embryo hatch and genetic background determines the hatch mechanism}

In our present study, markedly higher hatch rate observed in parthenogenetic embryos than fertilized embryos, regardless of oxygen concentration, resembled the result as previous reported [12]. Furthermore, when we use living imaging to monitor the hatch process in vitro, we amazedly found that fertilized embryos showed a hatch pattern different from parthenogenetic embryos. Fertilized embryos usually started hatching earlier and spent about $42 \mathrm{~h}$ to complete hatching, which was nearly seven times longer than parthenogenetic embryos that initiate hatching after $96 \mathrm{~h}$. This result indicated that these two types of embryos had totally different mechanisms to regulate hatch, which might be caused by the genetic background and ZP structure.

On the one hand, Parental genetic background difference can affect TE cells function and make 
parthenogenetic embryo have much more rapid expansion ability [12], which thus lead to such a short time hatched. On the other hand, there is at least one breach on the ZP of fertilized embryos due to sperm penetration, making them different that of parthenogenetic embryos. The hypotonic experiment result showed that the fertilized embryos prefer to start hatching from spermdriling gaps at about $72 \mathrm{~h}$ by plumping TE cells, thinning ZP and then breaking ZP, after which they continue to widen the ZP gap with "zona breaker" cells and the TE cells emerg to form dumb-bell shap like human embryos, and finally the whole embryo come out gradually $[52,53]$. Unlike fertilized embryos, parthenogenetic embryos have hermetical and intact ZP, and they usually keep plumping TE cells and thinning ZP till $96 \mathrm{~h}$ or later, and then suddenly break ZP and come out quickly.

To identify whether it is caused by the change of ZP integrity, we tried to make a breach on parthegenetic embryo's ZP artificially by Peizo micromanipulation [54]. No change happened when a tiny breach was made, whereas ZP-drilled parthenogenetic embryos started hatching at $96 \mathrm{~h}$ with a small mass of zona breaker cells when the breach size reached to $5 \mu \mathrm{m}$ which is as large as sperm penetration. Unexpectedly, instead of keeping emerging as fertilized embryos, these cells stoped and the whole embryo hatched suddently and quickly from another place of ZP after long-term expansion, just like normal parthenogenetic embryos. These results indicate that the divergence in ZP structure is not the main reason for the different hatching patterns between fertilized and parthenogenetic embryos. These data suggest that the sperm-drilling breach just induces embryo hatching but embryo itself knows how to continue this process by an unknown mechanism that requires further investigation.

\section{Conclusion}

In conclusion, the mechanism of mouse embryo hatch is regulated by both intrinsic and extrinsic factors in vitro. This result has significant clinical implications for the patients who requires AH during IVF treatment, meaning that, in addition to patients' clinical indications, the embryo culture condition should be taken into consideration, when this technique is used to facilitate embryo implantation. $\mathrm{O}_{2}$ concentration had different influences on the expression of key genes involving in MAPK pathway and superoxide dismutase family between fertilized and pharthenogenetic blastocysts. The regulation of gene expression might control embryonic cell proliferation and the allocation of ICM cells, indicated by the total embryonic cell number and ratio of Oct4-positive cells, and thus leads to diverse blastocyst hatch dynamics in different parental genetic background. Therefore, human embryos awaiting $\mathrm{AH}$ should be cultured under the atmospheric $\mathrm{O}_{2}$ (20\%) after fertilization, aiming to promote hatching and improve the reproductive outcomes, if the result of mouse study can be applied to humans. However, more animal and human clinical studies are required to clarify the mechanism underlying embryo hatching.

\section{Additional files}

Additional file 1: Real time PCR primers for genes expression analysis of $96 \mathrm{~h}$ blastocysts. (DOC $41 \mathrm{~kb}$ )

Additional file 2: Hatch process of fertilized embryos in vitro. Fertilized embryos started hatching in $72 \mathrm{~h}$ and then spent about $42 \mathrm{~h}$ emerging from it gradually and slowly until completely hatched in 120 h. (MP4 1099 kb)

Additional file 3: Hatch process of parthenogenetic embryos in vitro. Parthenogenetic embryos kept expansion until broken out suddenly and quickly within approximately $6 \mathrm{~h}$ after culture for $96 \mathrm{~h}$. (MP4 283 kb)

Additional file 4: Hypotonicity of $48 \mathrm{~h}$ fertilized embryo in vitro. Cytoplasm came out from sperm-breach quickly after transferred into pure water. (MP4 333 kb)

Additional file 5: Hypotonicity of parthenogenetic embryo in vitro. No cytoplasm came out from parthenogenetic embryos after transferred into pure water. (MP4 341 kb)

Additional file 6: Hypotonicity of parthenogenetic embryo with $5 \mu \mathrm{m}$ breach drilled on zona pellucid in vitro. Cytoplasm came out from artificial breach quickly as same as fertilized embryos after transferred into pure water. (MP4 330 kb)

Additional file 7: Hatch process of parthenogenetic embryo with $5 \mu \mathrm{m}$ breach drilled on zona pellucid in vitro. Parthenogenetic embryos started hatching slightly from artificial breach in 96 h, but embryonic cells did not keep emerge from it. They kept expansion and broke out from another place on zona pellucid in a short time as normal parthenogenetic embryos did. (MP4 580 kb)

\section{Abbreviations}

AH: Assisted hatching; ANOVA: One-way analysis of variance; ESCs: Embryonic stem cells; hCG: Human chorionic gonadotrophin; HKSOM: KSOM containing 14 mM Hepes and 4 mM sodium bicarbonate; ICM: Inner cell mass; IF: Immunofluorescence; KSOM: Potassium simplex optimized medium; KSOM AA: KSOM supplemented with nonessential and essential amino acids and $2.5 \mathrm{mM}$ hepes; MAPK: Mitogen-activated protein kinase; NAC: N-Acety-L-Cysteine; $\mathrm{O}_{2}$ : Oxygen; PBS-PVP: Phosphate buffered saline containing $0.1 \%$ polyvinyl pyrrolidone; PCR: Polymerase chain reaction; PLSD: Fisher's protected least-significant difference; ROS: Reactive oxygen species; SODs: Superoxide dismutases; SPF: Specific pathogen-free; TE: Trophectoderm; ZP: Zona pellucida

\section{Funding}

This work was supported by the National Key R\&D Program of China (2017YFC1001901 and 2017YFA0102801); National Natural Science Foundation of China (31671540, 81771579 and 81501327); Guangdong Science and Technology Department Planning Project (2015B020228002); and Science and Technology Planning Project of Guangdong Province (Grant No. 2014A020213017).

\section{Availability of data and materials}

The datasets analysed during the current study are available from the corresponding author on reasonable request.

\section{Authors' contributions}

$J \mathrm{H}$ conceived the study. $\mathrm{JH}$ and $\mathrm{HZ}$ designed the overall experimental scheme. SZ, SC, YS, LL, CD, and HD executed the experiments. SZ, CS, HZ and $\mathrm{JH}$ analyzed the data. SZ, $\mathrm{HZ}$ and $\mathrm{JH}$ wrote the paper. All authors read and approved the final manuscript. 


\section{Ethics approval and consent to participate}

All experimental protocols involving the handling of mice were approved by the Institutional Animal Care and Use Committee of Sun Yat-sen University (Approval No. IACUC-2014-0102), People's Republic of China.

\section{Competing interests}

The authors declare that they have no competing interests.

\section{Publisher's Note}

Springer Nature remains neutral with regard to jurisdictional claims in published maps and institutional affiliations.

\begin{abstract}
Author details
${ }^{1}$ Center for Reproductive Medicine, Key Laboratory for Reproductive Medicine of Guangdong Province, Key Laboratory for Major Obstetric Diseases of Guangdong Province, and Key Laboratory for Reproduction and Genetics of Guangdong Higher Education Institutes, the Third Affiliated Hospital of Guangzhou Medical University, Guangzhou 510150, China. ${ }^{2}$ Beijing Acorndx Biotechnology Co. Ltd, Beijing 100176, China. ${ }^{3}$ Key Laboratory of Reproductive Medicine of Guangdong Province, School of Life Sciences and the First Affiliated Hospital, Sun Yat-sen University, Guangzhou 510275, China.
\end{abstract}

Received: 30 December 2017 Accepted: 16 April 2018 Published online: 21 April 2018

\section{References}

1. Soupart P, Strong PA. Ultrastructural observations on polyspermic penetration of zona pellucida-free human oocytes inseminated in vitro. Fertil Steril. 1975;26:523-37.

2. O'Sullivan CM, Rancourt SL, Liu SY, Rancourt DE. A novel murine tryptase involved in blastocyst hatching and outgrowth. Reproduction. 2001;122:61-71.

3. Wassarman PM, Litscher ES. Mammalian fertilization: the egg's multifunctional zona pellucida. Int J Dev Biol. 2008:52:665-76.

4. Cohen J, Elsner C, Kort H, Malter H, Massey J, Mayer MP, et al. Impairment of the hatching process following IVF in the human and improvement of implantation by assisting hatching using micromanipulation. Hum Reprod. 1990:5:7-13.

5. McLaren A. Can mouse blastocysts stimulate a uterine response before losing the zona pellucida? J Reprod Fertil. 1969;19:199-201.

6. Carlone DL, Skalnik DG. CpG binding protein is crucial for early embryonic development. Mol Cell Biol. 2001;21:7601-6.

7. Chen HW, Chen JJ, Yu SL, Li HN, Yang PC, Su CM, et al. Transcriptome analysis in blastocyst hatching by cDNA microarray. Hum Reprod. 2005:20:2492-501.

8. Gwatkin RB, Williams DT, Hartmann JF, Kniazuk M. The zona reaction of hamster and mouse eggs: production in vitro by a trypsin-like protease from cortical granules. J Reprod Fertil. 1973;32:259-65.

9. Harvey AJ, Kind KL, Pantaleon M, Armstrong DT, Thompson JG. Oxygen-regulated gene expression in bovine blastocysts. Biol Reprod. 2004;71:1108-19.

10. Huang JC, Wun WS, Goldsby JS, Wun IC, Falconi SM, Wu KK. Prostacyclin enhances embryo hatching but not sperm motility. Hum Reprod. 2003;18:2582-9.

11. Kelleher AM, Peng W, Pru JK, Pru CA, DeMayo FJ, Spencer TE. Forkhead box a2 (FOXA2) is essential for uterine function and fertility. Proc Natl Acad Sci U S A. 2017;114:E1018-E26.

12. Latham KE, Kutyna K, Wang Q. Genetic variation in trophectoderm function in parthenogenetic mouse embryos. Dev Genet. 1999:24:329-35.

13. Montag $M$, Koll $B$, Holmes $P$, van der $V$. Significance of the number of embryonic cells and the state of the zona pellucida for hatching of mouse blastocysts in vitro versus in vivo. Biol Reprod. 2000;62:1738-44.

14. Qin J, Diaz-Cueto L, Schwarze JE, Takahashi Y, Imai M, Isuzugawa K, et al. Effects of progranulin on blastocyst hatching and subsequent adhesion and outgrowth in the mouse. Biol Reprod. 2005;73:434-42.

15. Pool TB. An update on embryo culture for human assisted reproductive technology: media, performance, and safety. Semin Reprod Med. 2005;23:309-18.

16. Feil D, Lane M, Roberts $C T$, Kelley RL, Edwards $L J$, Thompson JG, et al. Effect of culturing mouse embryos under different oxygen concentrations on subsequent fetal and placental development. J Physiol. 2006;572:87-96.
17. Fischer-Brown A, Monson R, Parrish J, Rutledge J. Cell allocation in bovine embryos cultured in two media under two oxygen concentrations. Zygote. 2002;10:341-8.

18. Harlow GM, Quinn P. Foetal and placental growth in the mouse after pre-implantation development in vitro under oxygen concentrations of 5 and 20\%. Aust J Biol Sci. 1979:32:363-9.

19. Karagenc L, Sertkaya Z, Ciray N, Ulug U, Bahceci M. Impact of oxygen concentration on embryonic development of mouse zygotes. Reprod BioMed Online. 2004:9:409-17.

20. Karja NW, Wongsrikeao P, Murakami M, Agung B, Fahrudin M, Nagai T, et al. Effects of oxygen tension on the development and quality of porcine in vitro fertilized embryos. Theriogenology. 2004;62:1585-95.

21. Lash GE, Otun HA, Innes BA, Bulmer JN, Searle RF, Robson SC. Low oxygen concentrations inhibit trophoblast cell invasion from early gestation placental explants via alterations in levels of the urokinase plasminogen activator system. Biol Reprod. 2006;74:403-9.

22. Quinn P, Harlow GM. The effect of oxygen on the development of preimplantation mouse embryos in vitro. J Exp Zool. 1978;206:73-80.

23. Rho GJ, Kim DS, Son WJ, Cho SR, Kim JG, et al. Influence of in vitro oxygen concentrations on preimplantation embryo development, gene expression and production of Hanwoo calves following embryo transfer. Mol Reprod Dev. 2007;74:486-96.

24. Umaoka Y, Noda Y, Narimoto K, Mori T. Effects of oxygen toxicity on early development of mouse embryos. Mol Reprod Dev. 1992;31:28-33.

25. Yuan YQ, Van Soom A, Coopman FO, Mintiens K, Boerjan ML, Van Zeveren A et al. Influence of oxygen tension on apoptosis and hatching in bovine embryos cultured in vitro. Theriogenology. 2003;59:1585-96.

26. Dumoulin JC, Vanvuchelen RC, Land JA, Pieters MH, Geraedts JP, Evers JL. Effect of oxygen concentration on in vitro fertilization and embryo culture in the human and the mouse. Fertil Steril. 1995;63:115-9.

27. Dumoulin JC, Meijers CJ, Bras M, Coonen E, Geraedts JP, Evers JL. Effect of oxygen concentration on human in-vitro fertilization and embryo culture. Hum Reprod. 1999;14:465-9.

28. Kind KL, Collett RA, Harvey AJ, Thompson JG. Oxygen-regulated expression of GLUT-1, GLUT-3, and VEGF in the mouse blastocyst. Mol Reprod Dev. 2005;70:37-44.

29. Liu L, Ju JC, Yang X. Parthenogenetic development and protein patterns of newly matured bovine oocytes after chemical activation. Mol Reprod Dev. 1998:49:298-307.

30. Liu L, Trimarchi JR, Keefe DL. Haploidy but not parthenogenetic activation leads to increased incidence of apoptosis in mouse embryos. Biol Reprod. 2002;66:204-10

31. Cheeseman LP, Boulanger J, Bond LM, Schuh M. Two pathways regulate cortical granule translocation to prevent polyspermy in mouse oocytes. Nat Commun. 2016:7:13726.

32. Bianchi E, Wright GJ. Sperm meets egg: the genetics of mammalian fertilization. Annu Rev Genet. 2016:50:93-111.

33. Okabe M. The cell biology of mammalian fertilization. Development. 2013:140:4471-9.

34. Wassarman P, Chen J, Cohen N, Litscher E, Liu C, Qi H, et al. Structure and function of the mammalian egg zona pellucida. J Exp Zool. 1999;285:251-8.

35. Huang J, Okuka M, McLean M, Keefe DL, Liu L. Telomere susceptibility to cigarette smoke-induced oxidative damage and chromosomal instability of mouse embryos in vitro. Free Radic Biol Med. 2010;48:1663-76.

36. Huang J, Okuka M, McLean M, Keefe DL, Liu L. Effects of cigarette smoke on fertilization and embryo development in vivo. Fertil Steril. 2009;92:1456-65.

37. Liu J, Liu M, Ye X, Liu K, Huang J, Wang L, et al. Delay in oocyte aging in mice by the antioxidant N-acetyl-L-cysteine (NAC). Hum Reprod. 2012;27:1411-20.

38. Sakao Y, Kawai T, Takeuchi O, Copeland NG, Gilbert DJ, Jenkins NA, et al. Mouse proteasomal ATPases Psmc3 and Psmc4: genomic organization and gene targeting. Genomics. 2000;67:1-7.

39. Maekawa M, Yamamoto T, Kohno M, Takeichi M, Nishida E. Requirement for ERK MAP kinase in mouse preimplantation development. Development. 2007;134:2751-9.

40. Goto $Y$, Noda $Y$, Narimoto $K$, Umaoka Y, Mori T. Oxidative stress on mouse embryo development in vitro. Free Radic Biol Med. 1992;13:47-53.

41. Orsi NM, Leese HJ. Protection against reactive oxygen species during mouse preimplantation embryo development: role of EDTA, oxygen tension, catalase, superoxide dismutase and pyruvate. Mol Reprod Dev. 2001;59:44-53. 
42. Genbacev OD, Prakobphol A, Foulk RA, Krtolica AR, llic D, Singer MS, et al. Trophoblast L-selectin-mediated adhesion at the maternal-fetal interface. Science. 2003;299:405-8.

43. Thouas GA, Dominguez F, Green MP, Vilella F, Simon C, Gardner DK. Soluble ligands and their receptors in human embryo development and implantation. Endocr Rev. 2015;36:92-130.

44. Gonzales DS, Bavister BD. Zona pellucida escape by hamster blastocysts in vitro is delayed and morphologically different compared with zona escape in vivo. Biol Reprod. 1995;52:470-80.

45. Schimmel T, Cohen J, Saunders H, Alikani M. Laser-assisted zona pellucida thinning does not facilitate hatching and may disrupt the in vitro hatching process: a morphokinetic study in the mouse. Hum Reprod. 2014;29:2670-9.

46. Park SB, Kim HJ, Choi YB, Ahn KH, Lee KH, Yang JB, et al. The effect of various assisted hatching techniques on the mouse early embryo development. Clin Exp Reprod Med. 2014:41:68-74.

47. Martins WP, Rocha IA, Ferriani RA, Nastri CO. Assisted hatching of human embryos: a systematic review and meta-analysis of randomized controlled trials. Hum Reprod Update. 2011;17:438-53.

48. Bavister B. Oxygen concentration and preimplantation development. Reprod BioMed Online. 2004;9:484-6.

49. Harvey AJ. The role of oxygen in ruminant preimplantation embryo development and metabolism. Anim Reprod Sci. 2007:98:113-28.

50. Liu L, Czerwiec E, Keefe DL. Effect of ploidy and parental genome composition on expression of Oct-4 protein in mouse embryos. Gene Expr Patterns. 2004;4:433-41.

51. Liu M, Yin Y, Ye X, Zeng M, Zhao Q Keefe DL, et al. Resveratrol protects against age-associated infertility in mice. Hum Reprod. 2013;28:707-17.

52. Cohen J, Feldberg D. Effects of the size and number of zona pellucida openings on hatching and trophoblast outgrowth in the mouse embryo. Mol Reprod Dev. 1991;30:70-8.

53. Sathananthan $\mathrm{H}$, Menezes J, Gunasheela S. Mechanics of human blastocyst hatching in vitro. Reprod BioMed Online. 2003;7:228-34.

54. Huang J, Deng K, Wu H, Liu Z, Chen Z, Cao S, et al. Efficient production of mice from embryonic stem cells injected into four- or eight-cell embryos by piezo micromanipulation. Stem Cells. 2008:26:1883-90.

\section{Ready to submit your research? Choose BMC and benefit from}

- fast, convenient online submission

- thorough peer review by experienced researchers in your field

- rapid publication on acceptance

- support for research data, including large and complex data types

- gold Open Access which fosters wider collaboration and increased citations - maximum visibility for your research: over $100 \mathrm{M}$ website views per year

At BMC, research is always in progress.

Learn more biomedcentral.com/submissions 